\title{
Effect of Proprioceptive Neuromuscular Facilitation Stretching on Pain, Hip Joint Range of Motion, and Functional Disability in Patients with Chronic Low Back Pain
}

\author{
Beomryong Kim ${ }^{a}$, Taewoo Kang ${ }^{b} \oplus$, Dahee Kim ${ }^{c}$

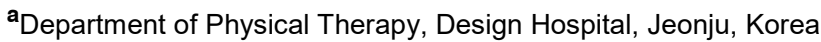 \\ ${ }^{b}$ Department of Physical Therapy, College of Health and Welfare, Woosuk University, Wanju, Korea \\ 'Department of Physical Therapy, Howon University, Gunsan, Korea
}

Objective: We aimed to identify the effects of proprioceptive neuromuscular facilitation (PNF) stretching on pain, hip range of motion, and functional disability in patients with chronic low back pain.

Design: Randomized controlled trial

Methods: In total, 45 patients with chronic low back pain were randomly divided into a conventional stretching group ( $\mathrm{n}=22)$ and a PNF stretching group $(\mathrm{n}=23)$. Both interventions were performed three times per week for 6 weeks. Assessments were made using the visual analog scale, Flexion-Abduction-External Rotation test, modified Thomas test, prone hip extension test, and Oswestry disability index before and after the 6-week intervention period. We conducted a paired t-test to compare the within-group findings before and after the intervention. An independent t-test was used to compare the between-group differences. The statistical significance level was set at $\alpha=0.05$, for all variables.

Results: Both groups showed significant improvements in pain, hip range of motion, and functional disability after the intervention $(\mathrm{p}<0.05)$. A significant difference was observed in pain, hip range of motion, and functional disability in patients belonging to the PNF stretching group $(\mathrm{p}<0.05)$.

Conclusions: This study provides evidence that the application of PNF stretching can effectively reduce pain and improve hip range of motion and functional disability in patients with chronic low back pain.

Key Words: Chronic low back pain, Functional disability, Hip range of motion, Proprioceptive neuromuscular facilitation

\section{Introduction}

Iliopsoas is a compound muscle which consists of the psoas major and iliacus. The psoas major is placed lateral to the vertebral column. It begins at the thoracic vertebrae 12 and the vertebral body of the lateral surface of the lumbar vertebrae 5 and extends to the transverse process of the lumbar vertebrae 1 to 5 , and finally attaches to the femur lesser trochante and the linea aspera medial. The iliacus has several points of origin; it starts with the iliac crest, anterior inferior iliac spine, iliolumbar ligament, and anterior sacroiliac ligament, and eventually attaches to femur lesser trochante and linea aspera medial [1]. In modern times, most of the day to day activities like work or study involves constant sitting or standing with limited bodily movements. Such conditions in the long-term can have a negative effect on the iliopsoas muscle and often lead to shortening of adaptations [2]. Since the iliopsoas is constantly active while sitting or standing, it plays an important role in stabilizing the pelvis and lumbar region along with the erector spinae and

Received: Jun 14, 2021 Revised: Jun 19, 2021 Accepted: Jun 20, 2021

Corresponding author: Dahee Kim (ORCID https://orcid.org/0000-0001-5455-5616)

Department of Physical Therapy, Howon University

64, Howondae 3-gil, Impi-myeon, Gunsan-si, Jeollabuk-do, Republic of Korea [54058]

Tel: + 82-63-450-7790 Fax: + 82-63-450-7799 E-mail: dream4656@naver.com

This is an Open-Access article distributed under the terms of the Creative Commons Attribution Non-Commercial License (http://creativecommons.org/licenses/ by-nc/4.0) which permits unrestricted non-commercial use, distribution, and reproduction in any medium, provided the original work is properly cited.

Copyright @ 2021 Korean Academy of Physical Therapy Rehabilitation Science 
quadratus lumborum [3]. Hence shortening or straining of the iliopsoas can cause excessive pelvic anterior tilt or increased spine extension during hip joint motion, thereby acting as a risk factor for low back pain [4].

Numerous studies have focused on the relaxation and elongation of the iliopsoas using relaxation techniques like massages and stretching. In a recent interventional study for shortened iliopsoas in patients with low back pain, Lee and Song [4] demonstrated that passive and active stretching can significantly rescue the length of iliopsoas, thereby reducing low back pain. Volpato et al. [5] had reported a reduced low back pain and improved flexibility while applying iliopsoas intervention with stabilization exercises. Lee et al. [6] showed significant changes in the thickness, muscle tone, and pelvic angle of the iliopsoas after deep muscle massage, passive stretching, and muscle energy technique intervention in patients with nonspecific low back pain.

Proprioceptive neuromuscular facilitation (PNF) is a form of flexibility exercises used to resolve muscle shortening and strain [7,8]. The "PNF stretching" is called "muscle energy techniques", "active musculature relaxation techniques", "rapid resistance duction", "active stretching", and "PNF stretching" depending on the group used or called [9]. Jeong and Kim [10] observed that application of PNF stretching significantly reduces low back pain and dysfunction in patients with chronic low back pain. Birinci et al. [11] reported significant improvements in elbow pain, range of motion (ROM), and arm function upon the application of PNF stretching in patients with elbow stiffness. Gunn et al. [12] reported that PNF stretching is more effective for hamstring flexibility than static stretching. Malai et al. [13] reported that PNF stretching immediately reduced low back pain, decreased lumbar lordosis angle, and increased transversus abdominis activation.

Hence PNF stretching has been identified as an effective method for increasing flexibility and reducing pain and dysfunction. And it can be easily used to treat patients who experience chronic low back pain as a result of limited hip joint motion. Nevertheless, previous studies using PNF stretching for chronic low back pain fail to address hip ROM and iliopsoas dysfunction. In addition, it has been addressed in previous studies, many studies conducted with increased reliability through randomization of long-term intervention of 6 weeks are hard to find. In this study, we investigated the effect of PNF stretching on pain, hip ROM, and dysfunction in patients with chronic low back pain and presented our findings as base data for intervention of patients with chronic low back pain.

\section{Materials and methods}

\section{Research design}

This was a single-blinded, randomized clinical trial that included 50 participants who were randomly assigned to two groups: The PNF and/or conventional stretching groups. The visual analog scale (VAS), Flexion-Abduction-External Rotation test (FABERT), modified Thomas test (MTT), prone hip extension test (PHET), and Oswestry disability index (ODI) results were identified as the primary results of this study. Concealed allocation was performed using GraphPad software prior to data collection by a qualified examiner. The examination was performed by an independent physiotherapist who was blinded to the identity of each group as well as patient's clinical information. And the observations were made at the beginning of the intervention and 6 weeks after the intervention. The examiner was provided with a separate examination form for documenting the results. Both PNF and conventional stretching interventions were applied by the same physiotherapist and were excluded from the outcome assessment (Figure 1).

\section{Participants}

The appropriate number of participants for this study was analyzed using the G-Power software program (G-Power software 3.1.2, University of Kiel, Germany). The number of samples required to maintain an actual power of 0.87 at a significant $\alpha$ level (0.05), a large effect size (0.8), and power (0.8) in the independent t-test was found to be 26 participants in each group.

This study was conducted at Design Hospital in Jeonju city between December 2019 and March 2020. Forty-five patients with chronic low back pain (CLBP) agreed to participate in the study. These participants were randomly assigned to the PNF and/or conventional stretching groups. The inclusion criteria for participation were as follows: (a) previously diagnosed with CLBP, 


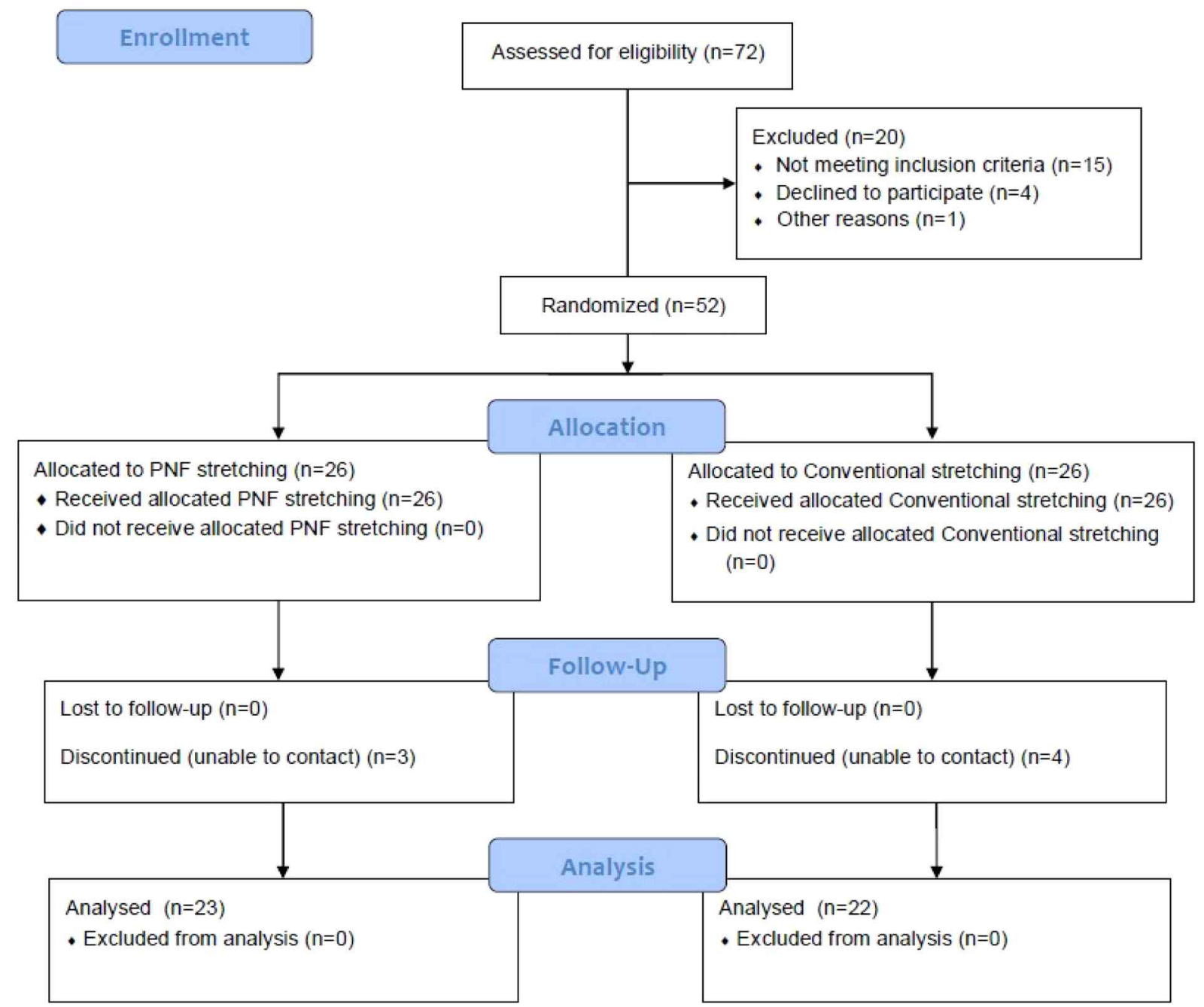

Figure 1. Flow chart of the study.

PNF: proprioceptive neuromuscular facilitation.

(b) pain in the lumbar spine for a period longer than 3 months, (c) pain with a severity level $>3$ as per VAS (0-10 cm; 0 no pain, 10 severe pain); and (d) a MTT result with an angle of $5^{\circ}$ or more. The exclusion criteria were as follows: (1) history of spinal and hip surgery; (2) previous record of spinal and hip fractures; (3) episodes of spinal and hip inflammation; (4) history of spondylolisthesis or spondylolysis; (5) history of rheumatoid arthritis, ankylosing spondylitis, or others; (6) persistent severe and acute pain; (7) history of neurological, respiratory, and cardiac disorders; (8) pregnancy; (9) osteoporosis; (10) continuous use of pain medications; and (11) mental problems or reduced cognitive ability. General characteristics of the participants' are presented in Table 1.

\section{Outcome measurements}

The degree of LBP was evaluated using the VAS, which provides a visual representation of the patient's pain. The degree of pain that the patient experienced was subjective and scaled from 0 to 10 . With 0 being the state of no pain at all and 10 being the state of extreme pain. The VAS has a high intra-rater reliability (intra-class correlation coefficient $[\mathrm{ICC}]=1.00$ ) and inter-rater reliability $(\mathrm{ICC}=0.99)$ [14].

The participants were in the supine position for the FABERT. For this exercise, the left hip was placed between the right thigh and knee to allow flexion, abduction, and external rotation of the left hip joint. The examiner stabilized the pelvis by applying pressure 
Table 1. General characteristics of the participants

$(\mathrm{n}=45)$

\begin{tabular}{lllll}
\hline Variables & All patients & PNF stretching & Conventional stretching & $\mathbf{p}^{\text {a) }}$ \\
\hline Number of patients & 45 & 23 & 22 & - \\
Sex (male/female) & $24 / 21$ & $13 / 10$ & $11 / 11$ & - \\
Age (y) & $45.13(12.71)$ & $45.13(12.69)$ & $45.14(13.03)$ & 0.255 \\
Height (cm) & $168.53(8.35)$ & $168.52(8.42)$ & $168.54(8.47)$ & 0.188 \\
Body weight (kg) & $66.07(10.17)$ & $66.78(10.37)$ & $65.32(10.14)$ & 0.194 \\
Body mass index (kg/cm $\left.{ }^{2}\right)$ & $23.09(1.47)$ & $23.34(1.51)$ & $22.82(1.41)$ & 0.060 \\
Non-dominant side (right/left) & $19 / 26$ & $10 / 13$ & $9 / 13$ & - \\
Visual analog scale (score) & $6.00(1.13)$ & $5.96(1.11)$ & $6.04(1.17)$ & $18.86(2.95)$ \\
FABER test (degree) & $19.35(2.70)$ & $19.83(2.40)$ & $18.04(6.39)$ & 0.093 \\
Modified Thomas test (degree) & $17.60(6.51)$ & $17.17(6.74)$ & $7.95(2.01)$ & 0.509 \\
Prone hip extension test (degree) & $7.67(2.13)$ & $7.39(2.25)$ & $54.45(6.47)$ & 0.335 \\
Oswestry disability index (score) & $54.75(6.73)$ & $55.04(7.11)$ & 0.327
\end{tabular}

Values are presented as mean (SD)

PNF: proprioceptive neuromuscular facilitation, FABER: Flexion-Abduction-External Rotation.

a) Shapiro-Wilk test

$* \mathrm{p}<0.05$

to the anterior-superior iliac spine. The left knee of the participant was slowly lowered toward the table by a passive force. The examiner measured the angle by placing a digital clinometer at the distal end of the medial epicondyle. The FABERT has a high intra-rater reliability $(\mathrm{ICC}=0.91)$ [15].

The MTT measurement allowed the participant to sit on the end of the table and then hold the knee and lie back. The examiner then instructed the participant to flex the knee and pull it closely to the chest area and hold it tightly. The leg to be measured was manually lowered to the table. The examiner then placed the knee joint in a flexion position of approximately $90^{\circ}$ and confirmed that the thigh was fully relaxed. The digital clinometer was then placed at the mid-point of the thigh, and the angle of the hip extension was measured. The MTT has a high inter-rater reliability $(\mathrm{ICC}=0.89-0.92)$ [16].

The PHET measurement was performed with the participant in a prone position with the arm placed next to the torso. The examiner then instructed the participant to actively maximize the extension of the hip joint. A digital clinometer was placed on the thigh, above the popliteal and the angle of hip extension was measured. PHET has inter-rater reliability $(\mathrm{ICC}=0.76)$, sensitivity (0.18-0.27), and specificity (0.63-0.78) [17].

The ODI was proposed to measure the degree of disability that occurs due to LBP. It addresses 10 questions (pain intensity, personal care, lifting, sitting, standing, walking, sleeping, sex life, and social life). Depending on the potential of the task, scores are provided between 0 to 5 : the higher the score, the larger the disability [18]. The ODI is calculated by dividing the complete score by the number of questions and then multiplying by 100 .

\section{Intervention and procedure}

All groups received 10-minutes of trunk stabilization exercise and 40 minutes of general physical therapy. The PNF stretching group received an additional 20 minutes of iliopsoas stretching using PNF techniques. The conventional stretching group received an additional 20-minutes of iliopsoas stretching using conventional techniques. All interventions were conducted three times per week for 6 weeks.

General physical therapy consisted of 15 minutes of hot pack treatment (two to three sheets of towels at $80^{\circ} \mathrm{C}$ ), 15 minutes of interferential current therapy $(100 \mathrm{~Hz}$ of 


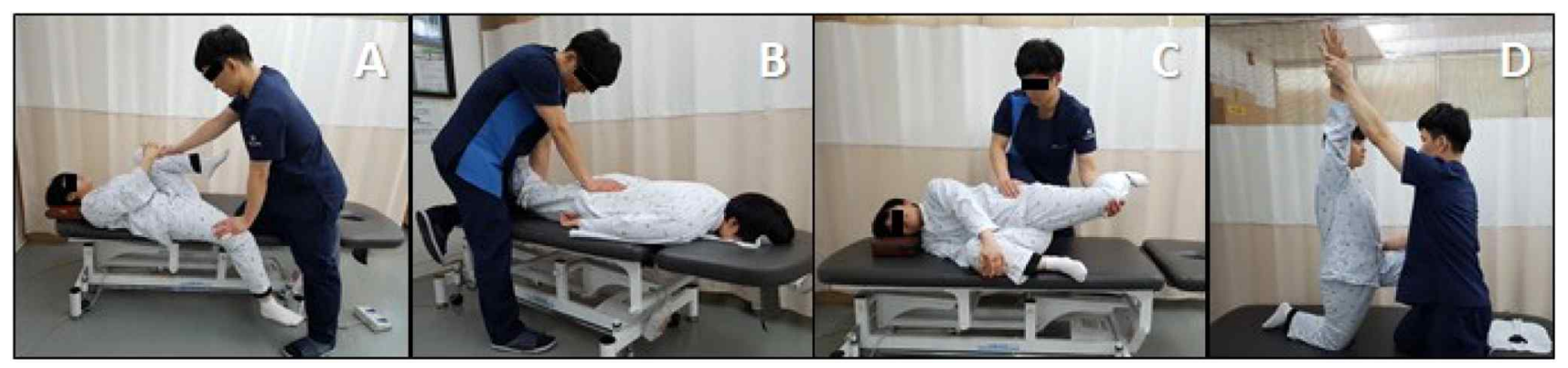

Figure 2. Proprioceptive neuromuscular facilitation iliopsoas stretching. (A) supine. (B) prone. (C) side-lying. (D) half-kneeling positions.

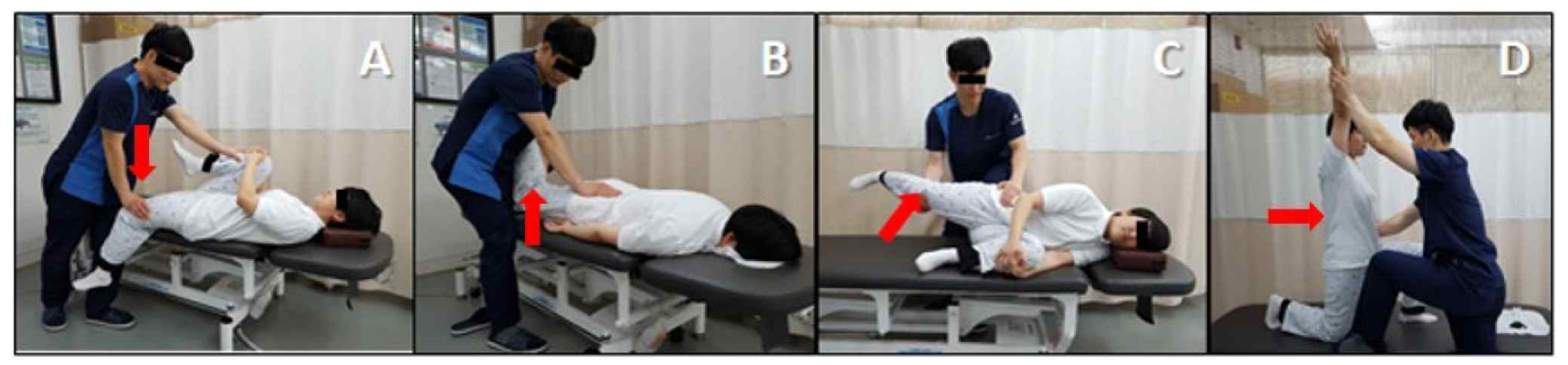

Figure 3. Conventional iliopsoas stretching. (A) supine. (B) prone. (C) side-lying. (D) half-kneeling positions.

constant current), and 10 minutes of ultrasound ( $0.75 \mathrm{MHz}$, continuous wave) [19]. The trunk stabilization exercise was performed by 10 sets of bracing and bridge exercises, respectively with 30 -second hold and 10-seconds rest [20].

PNF stretching was performed using contract-relax techniques of agonists in supine, prone, side-lying, and half-kneeling positions [21]. Contract-relax was applied with a 6-second contraction with $80 \%$ force of the maximal isometric contraction on the iliopsoas followed by a 15 -second passive static stretching in the opposite direction of the iliopsoas [7]. Three sets of stretching were performed for each position. The PNF iliopsoas stretching is shown in Figure 2.

Conventional stretching was also performed by passive static stretching in the supine, prone, side-lying, and half-kneeling positions. Passive static stretching of the iliopsoas was performed for 30 seconds at the maximum stretch position [20]. Three sets of stretching were conducted for each position and are demonstrated in Figure 3.

\section{Statistical analysis}

Statistical analysis was performed using the statistical program for Windows; the SPSS/PC Statistics software (version 23.0, IBM Co., USA). The Shapiro-Wilk test was used to check the normal distribution of the data. A paired t-test was performed to compare differences in pain and hip ROM within the groups before and after the intervention. An independent t-test was performed to compare the differences between the PNF and conventional stretching groups. Statistical significance was set at a P-value of less than 0.05 .

\section{Results}

\section{Comparison of the VAS score changes}

Within-group changes in the VAS scores showed significant differences between the PNF $(t=29.667, \mathrm{p}<0.05)$ and conventional stretching groups $(\mathrm{t}=32.078, \mathrm{p}<0.05)$. The between-group changes in the VAS scores after intervention also showed significant differences between the PNF and conventional stretching groups $(\mathrm{t}=2.163 ; \mathrm{p}<0.05 ; 95 \%$ confidence interval $[\mathrm{CI}], 0.02$ to 0.71 ) (Table 2).

\section{Comparison of FABERT score changes}

Within-group changes in the FABERT scores were significantly different between the PNF $(t=58.874, p<0.05)$ 
Table 2. Comparison of variable within and between groups

\begin{tabular}{|c|c|c|c|c|c|c|c|c|}
\hline \multirow{2}{*}{ Variables } & & \multirow{2}{*}{$\begin{array}{l}\text { PNF } \\
\text { stretching } \\
(\mathbf{n}=\mathbf{2 3})\end{array}$} & \multirow{2}{*}{$\begin{array}{l}\text { Conventional } \\
\text { stretching } \\
(n=22)\end{array}$} & \multicolumn{2}{|c|}{ Difference (post-pre) } & \multirow[b]{2}{*}{$95 \% \mathrm{CI}$} & \multirow[b]{2}{*}{$\mathbf{t}$} & \multirow[b]{2}{*}{$\mathbf{p}^{\text {b) }}$} \\
\hline & & & & $\begin{array}{l}\text { PNF } \\
\text { stretching }\end{array}$ & $\begin{array}{l}\text { Conventional } \\
\text { stretching }\end{array}$ & & & \\
\hline \multirow{4}{*}{$\begin{array}{l}\text { Visual analog } \\
\text { scale (score) }\end{array}$} & Pre & $5.96(1.11)$ & $6.04(1.17)$ & \multirow{4}{*}{$\begin{array}{l}3.87 \\
(0.62)\end{array}$} & \multirow{4}{*}{$\begin{array}{l}3.50 \\
(0.51)\end{array}$} & \multirow{4}{*}{$\begin{array}{l}0.02 \text { to } \\
0.71\end{array}$} & \multirow{4}{*}{2.163} & \multirow{4}{*}{$0.036^{*}$} \\
\hline & Post & $2.09(0.95)$ & $2.54(1.01)$ & & & & & \\
\hline & $\mathrm{t}$ & 29.667 & 32.078 & & & & & \\
\hline & $\mathrm{P}^{\text {a) }}$ & $0.000^{*}$ & $0.000^{*}$ & & & & & \\
\hline \multirow{4}{*}{$\begin{array}{l}\text { FABER test } \\
\text { (degree) }\end{array}$} & Pre & $19.83(2.40)$ & $18.86(2.95)$ & \multirow{4}{*}{$\begin{array}{l}9.61 \\
(0.78)\end{array}$} & \multirow{4}{*}{$\begin{array}{l}8.27 \\
(0.70)\end{array}$} & \multirow{4}{*}{$\begin{array}{l}0.89 \text { to } \\
1.78\end{array}$} & \multirow{4}{*}{6.016} & \multirow{4}{*}{$0.000^{*}$} \\
\hline & Post & $10.22(2.57)$ & $10.59(2.82)$ & & & & & \\
\hline & $\mathrm{t}$ & 58.874 & 55.235 & & & & & \\
\hline & $\mathrm{P}^{\text {a) }}$ & $0.000^{*}$ & $0.000^{*}$ & & & & & \\
\hline \multirow{4}{*}{$\begin{array}{l}\text { Modified Thomas } \\
\text { test (degree) }\end{array}$} & Pre & $17.17(6.74)$ & $18.04(6.39)$ & \multirow{4}{*}{$\begin{array}{l}10.39 \\
(1.85)\end{array}$} & \multirow{4}{*}{$\begin{array}{l}9.32 \\
(1.17)\end{array}$} & \multirow{4}{*}{$\begin{array}{l}0.14 \text { to } \\
2.01\end{array}$} & \multirow{4}{*}{2.311} & \multirow{4}{*}{$0.026^{*}$} \\
\hline & Post & $6.78(5.26)$ & $8.73(5.43)$ & & & & & \\
\hline & $\mathrm{t}$ & 26.905 & 37.339 & & & & & \\
\hline & $\mathrm{P}^{\text {a) }}$ & $0.000^{*}$ & $0.000^{*}$ & & & & & \\
\hline \multirow{4}{*}{$\begin{array}{l}\text { Prone hip } \\
\text { extension test } \\
\text { (degree) }\end{array}$} & Pre & $7.39(2.25)$ & $7.95(2.01)$ & \multirow{4}{*}{$\begin{array}{l}-5.69 \\
(1.33)\end{array}$} & \multirow{4}{*}{$\begin{array}{l}-4.73 \\
(1.35)\end{array}$} & \multirow{4}{*}{$\begin{array}{l}-1.77 \text { to } \\
-0.16\end{array}$} & \multirow{4}{*}{-2.423} & \multirow{4}{*}{$0.020^{*}$} \\
\hline & Post & $13.09(3.36)$ & $12.68(3.30)$ & & & & & \\
\hline & $\mathrm{t}$ & -20.550 & -16.405 & & & & & \\
\hline & $\mathrm{P}^{\text {a) }}$ & $0.000^{*}$ & $0.000^{*}$ & & & & & \\
\hline \multirow{4}{*}{$\begin{array}{l}\text { Oswestry } \\
\text { disability index } \\
\text { (score) }\end{array}$} & Pre & $55.04(7.11)$ & $54.45(6.47)$ & \multirow{4}{*}{$\begin{array}{l}31.74 \\
(2.65)\end{array}$} & \multirow{4}{*}{$\begin{array}{l}30.09 \\
(2.50)\end{array}$} & & & \\
\hline & Post & $23.30(4.88)$ & $24.36(5.22)$ & & & 0.10 to & 2.142 & $0.038^{*}$ \\
\hline & $\mathrm{t}$ & 57.451 & 56.334 & & & 3.20 & & \\
\hline & $\mathrm{P}^{\text {a) }}$ & $0.000^{*}$ & $0.000^{*}$ & & & & & \\
\hline
\end{tabular}

Values are presented as mean (SD)

PNF: proprioceptive neuromuscular facilitation, CI: confidence interval.

a) paired t-test, ${ }^{\text {b) }}$ independentt-test,

${ }^{*} \mathrm{p}<0.05$

and conventional stretching groups $(\mathrm{t}=55.235, \mathrm{p}<0.05)$. The between-group changes in the FABERT scores after intervention showed significant differences between the PNF and conventional stretching groups $(\mathrm{t}=6.016$; $\mathrm{p}<0.05 ; 95 \% \mathrm{CI}, 0.89$ to 1.78 ) (Table 2).

\section{Comparison of the MTT score changes}

Within-group changes in the MTT scores showed significant differences between the PNF $(t=26.905$, $\mathrm{p}<0.05)$ and conventional stretching groups $(\mathrm{t}=37.339$, $\mathrm{p}<0.05)$. The between-group changes in the MTT scores after intervention showed significant differences between the PNF and conventional stretching groups $(\mathrm{t}=2.311 ; \mathrm{p}<0.05 ; 95 \% \mathrm{CI}, 0.14$ to 2.01$)$ (Table 2).

\section{Comparison of the PHET score changes}

Within-group changes in the PHET scores were significantly different between the PNF $(t=-20.550, p<0.05)$ and conventional stretching groups $(t=-16.405, p<0.05)$. The between-group changes in the PHET scores after intervention showed significant differences between the PNF and conventional stretching groups $(\mathrm{t}=-2.423 ; \mathrm{p}<0.05$; $95 \%$ CI, -1.77 to -0.16 ) (Table 2). 


\section{Comparison of the ODI score changes}

Within-group changes in the ODI scores were significantly different between the PNF ( $t=57.451$, $\mathrm{p}<0.05)$ and conventional stretching groups $(\mathrm{t}=56.334$, $\mathrm{p}<0.05)$. The between-group changes in the ODI scores after intervention showed significant differences between the PNF and conventional stretching groups $(\mathrm{t}=2.142$; $\mathrm{p}<0.05 ; 95 \% \mathrm{CI}, 0.10$ to 3.20 ) (Table 2).

\section{Discussion}

The effects of PNF and conventional stretching on LBP (VAS), functional disability (ODI), and hip ROM (FABERT, MTT, PHET) are discussed below.

The iliopsoas is a major muscle of the hip aiding in its flexion and assisting in functions like posture, standing, sitting, and so on. A shortening of the iliopsoas muscle can result in anterior pelvic tilt and trunk extension, thus, leading to LBP [4]. In this study, PNF and conventional stretching were applied to patients who experienced LBP due to shortening of the iliopsoas. Although both methods of intervention showed improved results for the independent variables, PNF stretching showed a great recovery. Previously, Decicco and Fisher [22] had demonstrated PNF stretching on randomly allocated overhead throwing sport athletes along with control groups. After 6 weeks of training, the PNF stretching group showed significant improvement in external rotation. In another study, Puentedura et al. [23] applied PNF and static stretching on the hamstring of healthy adults. Although both the methods improved hamstring flexibility, there was no difference in the efficacy of the two methods. However, when Birinci et al. [11] randomly allocated PNF and static stretching to patients with elbow stiffness after a treated elbow fracture, they observed improvement in arm pain, ROM, and function particularly in the PNF stretching group. Tucker and Slone [24] applied PNF stretching, PNF stretching combined with vibration, and static stretching to patients with glenohumeral internal rotation deficit. Out of the three, PNF stretching in combination with vibration showed the greatest change in internal rotation. This results showed that in conventional stretching, parallel elastic components of muscle are stretching mainly. However, muscle contraction caused by PNF's Contract-relax technique is believed to have resulted in a relatively greater effect of simultaneously stretching parallel and serial elastic components [25]. Thus it is inferred that PNF stretching does not offer a greater advantage over conventional stretching in healthy adults but is found to be more effective than conventional stretching in patients with rotational deficit. The outcomes of these foregoing studies support the findings in the present study.

LBP occurs as a result of weakening of muscles of the abdomen and buttocks, as well as the shortening of the iliopsoas. Dysfunction in the iliopsoas leads to excessive lumbar extension and pelvic anterior tilt, that later culminates to LBP [26]. In this study, both PNF and conventional stretching groups showed a decrease in LBP and functional disability. Moreover, the PNF stretching group showed a significant decrease in LBP and functional disability as compared to the conventional stretching group. Interventions are mostly focused on core stabilized muscles that are weak in patients with LBP. However, it is also important to address the pain and ROM limitations that may occur in the shortened muscles [6]. Previously, Lee et al. [6] had performed iliopsoas relaxation and stretching in patients with nonspecific LBP. Likewise, in this study we reported a decrease in tension and thickness of the iliopsoas as well as a pelvic anterior tilt. Lee and Song [4] had also shown a significant decrease in pain after applying iliopsoas stretching to patients with LBP. These findings also support the results of the present study which claims that iliopsoas stretching reduces anterior pelvic tilt, increases abdominal and buttock muscle activity, and decreases LBP.

The FABERT attempts to reproduce pain in the hip, lumbar spine, and sacroiliac regions in order to confirm the pathology. This test is a manual test tool for musculoskeletal pathologies such as, hip, lumbar spine, sacroiliac joint dysfunction, and iliopsoas spasm [27]. In this study, both PNF and conventional stretching groups showed a decrease in the FABERT angle, where the PNF stretching group showed a more significant decrease in the FABERT angle than the conventional stretching group. Previously, Bagwell et al. [15] had performed the FABERT in healthy adults and found that the mean angle was $15.0^{\circ} \pm 7.6^{\circ}$. In 
this study we found that PNF stretching lowered the angle from $19.83 \pm 2.4^{\circ}$ to $10.22 \pm 2.57^{\circ}$, which is lower than the average angle of healthy adults. We also observed a change of angle post conventional stretching from $18.86^{\circ} \pm 2.95^{\circ}$ to $10.59^{\circ} \pm 2.82^{\circ}$, which is also lower than the average angle of healthy adults. The minimal detectable change for the FABERT angle was reported to be $6.1^{\circ}$ angle. This study showed a $9.61 \pm 0.78^{\circ}$ change in the angle after PNF stretching, and $8.27 \pm 0.70^{\circ}$ change after conventional stretching. These results are comparable to those of previous studies which show that PNF and conventional stretching can cause changes in the FABERT angle; likewise the FABERT may also be used as an iliopsoas test tool.

The MTT is used to evaluate the flexibility of the iliopsoas, rectus femoris, and tensor of fascia lata $[16,28]$. In this study, both PNF and conventional stretching groups showed a decrease in the MTT angle. Moreover, the decrease in the MTT angle was higher in the PNF stretching group than in the conventional stretching group. In another study, Kim et al. [29] had applied deep friction massage, stretching, and muscle energy technique for the elongation and relaxation of iliopsoas in 45 healthy adults. This resulted in the elongation and relaxation of the iliopsoas and led to the neutralization of the pelvic tilt. Similarly, Lee and Song [4] had also reported a reduction in the hip extension angle of the MTT after applying iliopsoas stretching on patients with LBP. These previous studies support the PNF and conventional stretching data shown in our study.

The PHET is a clinical test developed by Janda to evaluate exercise control and deficits in a variety of patients with musculoskeletal pain. During normal hip extension, movements are activated in the order of hamstring, gluteus maximus, opposite erector spinae, and same erector spinae respectively [30]. In this study, the angle of the PHET increased in both the PNF and conventional stretching groups. However, the PNF stretching group showed a more significant increase in the angle of the MTT than the conventional stretching group. Patients with LBP have a weakened hip extension [31]. The weakening of the hip extension increases the tension of the iliopsoas [32]. Kim et al. [29] reported a significant decrease in the tension of iliopsoas after applying iliopsoas stretching and relaxation techniques to healthy adults. In the current study, PNF and conventional stretching decreased the tension of the iliopsoas, and it was observed that the PHET score was increased, thereby providing a good environment to activate the hip extensor.

The length of iliopsoas was increased through PNF and conventional stretching in this study and therefore they was effective in changing LBP, hip ROM, and dysfunction. PNF and conventional stretching were also found to be important in preventing ROM and dysfunction. The PNF stretching group, in particular, showed a significantly lower LBP, hip ROM, and dysfunction than the conventional stretching group. These results suggest that iliopsoas stretching is crucial for patients with LBP. Therefore, PNF stretching is a more preferable than conventional stretching for patients with LBP in terms of better results. Our study is that PNF stretching was applied to LBP patients with the application of general physical therapy and trunk stabilization exercise. Hence, it is hard to showed the results of this study only by stretching the iliopsoas muscle.

\section{Conclusion}

In this study we have demonstrated that general physical therapy and PNF iliopsoas stretching were effective in reducing LBP, improving hip ROM, and decreasing dysfunction. Although general physical therapy and trunk stabilization exercises are sufficient for treating patients with LBP, iliopsoas intervention provides progressive results.

\section{Reference}

1. Cronin CG, Lohan DG, Meehan CP, Delappe E, McLoughlin R, O'Sullivan GJ, et al. Anatomy, pathology, imaging and intervention of the iliopsoas muscle revisited. EmergRadiol. 2008;15:295-310.

2. Akbari A, Mohammadi M. Effect of post isometric stretch duration on the hip joint extension in females with short iliopsoas muscle. J Shahrekord University Med Sci. 2007;9:68-75.

3. Penning L. Psoas muscle and lumbar spine stability: 
a concept uniting existing controversies. Eur Spine J. 2000;9:577-85.

4. Lee J, Song B. The effects of the passive and active stretching exercises of iliopsoas muscles on low back pain patients. J Spec Educ Rehabil Sci. 2015;54:291-307.

5. Volpato CP, Added MAN, Richter G, Tanaka V, Carvalho N, Freitas D. Influence of stretching and strengthening of the iliopsoas associated with lumbar segmental stabilization exercises in patients with low back pain: the pilot study. J Exerc Sports Orthop. 2014;25:27-32.

6. Lee HJ, Shim JH, Kim JW, Kim KS. The fusion effect of deep transverse stroking, manual stretching exercise and active muscle release technique on psoas major muslce thickness and muscle tone and pelvic angle of non-specific low back pain patient. J Korea Converg Soc. 2018;9:137-44.

7. Konrad A, Stafilidis S, Tilp M. Effects of acute static, ballistic, and PNF stretching exercise on the muscle and tendon tissue properties. Scand J Med Sci sports. 2017;27:1070-80.

8. Ryu BH. The effect of joint mobilization with PNF stretch exercise on ankle joint range of motion, plantar pressure, and balance in patients with stroke. J Int Acad Phys Ther Res. 2018;9:1642-50.

9. Chaitow L, Crenshaw K. Muscle energy techniques. 3rd ed. Amsterdam: Elsevier Health Sciences; 2006.

10. Jeong WM, Kim BR. The effects of proprioceptive neuromuscular facilitation exercise on the pain and functional disability index of patients with chronic lower back pain. PNF \& Mov. 2017;15:195-200.

11. Birinci T, Razak Ozdincler A, Altun S, Kural C. A structured exercise programme combined with proprioceptive neuromuscular facilitation stretching or static stretching in posttraumatic stiffness of the elbow: a randomized controlled trial. Clin Rehabil. 2019;33:241-52.

12. Gunn LJ, Stewart JC, Morgan B, Metts ST, Magnuson JM, Iglowski NJ, et al. Instrument-assisted soft tissue mobilization and proprioceptive neuromuscular facilitation techniques improve hamstring flexibility better than static stretching alone: a randomized clinical trial. J Man ManipTher. 2019;27:15-23.

13. Malai S, Pichaiyongwongdee S, Sakulsriprasert P.
Immediate Effect of Hold-Relax Stretching of Iliopsoas Muscle on Transversus Abdominis Muscle Activation in Chronic Non-Specific Low Back Pain with Lumbar Hyperlordosis. J Med Assoc Thai. 2015;98 Suppl 5:S6-11.

14. Wagner DR, Tatsugawa K, Parker D, Young TA. Reliability and utility of a visual analog scale for the assessment of acute mountain sickness. High Alt Med Biol. 2007;8:27-31.

15. Bagwell JJ, Bauer L, Gradoz M, Grindstaff TL. The reliability of FABER test hip range of motion measurements. Int J Sports Phys Ther. 2016;11:1101-5.

16. Clapis PA, Davis SM, Davis RO. Reliability of inclinometer and goniometric measurements of hip extension flexibility using the modified Thomas test. Physiother Theory Pract. 2008;24:135-41.

17. Bruno PA, Millar DP, Goertzen DA. Inter-rater agreement, sensitivity, and specificity of the prone hip extension test and active straight leg raise test. Chiropr Man Therap. 2014;22:23.

18. Fairbank JC. Oswestry disability index. J Neurosurg Spine. 2014;20:239-41.

19. Kim BR, Lee HJ. Effects of proprioceptive neuromuscular facilitation-based abdominal muscle strengthening training on pulmonary function, pain, and functional disability index in chronic low back pain patients. J Exer Rehabil. 2017;13:486-90

20. Kang TW, Kim BR. The effects of stretching and strengthening exercise on the pain, pelvic tilt, functional disability index, and balance ability of patients with chronic lower back pain. J Korean Phys Ther. 2019;31:7-12.

21. Adler SS, Beckers D, Buck M. PNF in practice: an illustrated guide. New York: Springer; 2007.

22. Decicco PV, Fisher MM. The effects of proprioceptive neuromuscular facilitation stretching on shoulder range of motion in overhand athletes. J Sports Med Phys Fitness. 2005;45:183-7.

23. Puentedura EJ, Huijbregts PA, Celeste S, Edwards D, In A, Landers MR, et al. Immediate effects of quantified hamstring stretching: hold-relax proprioceptive neuromuscular facilitation versus static stretching. Phys Ther Sport. 2011;12:122-6.

24. Tucker WS, Slone SW. The acute effects of hold-relax proprioceptive neuromuscular facilitation with vibration therapy on glenohumeral internal-rotation deficit. J 
Sport Rehabil. 2016;25:248-54.

25. Lederman E. The science \& practice of manual therapy. 2nd ed.Amsterdam: Elsevier Health Sciences; 2005.

26. Youdas JW, Garrett TR, Egan KS, Therneau TM. Lumbar lordosis and pelvic inclination in adults with chronic low back pain. Phys Ther. 2000;80:261-75.

27. Martin RL, Sekiya JK. The interrater reliability of 4 clinical tests used to assess individuals with musculoskeletal hip pain. J Orthop Sports Phys Ther. 2008;38:71-7.

28. Glard Y, Launay F, Viehweger E, Guillaume JM, Jouve JL, Bollini G. Hip flexion contracture and lumbar spine lordosis in myelomeningocele. J Ped Orthop. 2005;25:476-8.

29. Kim DW, Shim JH, Choung SD. The effect of deep friction massage, modified thomas's stretching and muscle energy technique on thickness of psoas major and pelvic angle. Arch Orthop Sports Phys Ther. 2016;12:1-7.

30. Frank C, Page P, Lardner R. Assessment and treatment of muscle imbalance: the Janda approach. 1st ed. Illinois: Human kinetics; 2009.

31. Nadler SF, Malanga GA, Feinberg JH, Prybicien M, Stitik TP, DePrince M. Relationship between hip muscle imbalance and occurrence of low back pain in collegiate athletes: a prospective study. Am J Phys Med Rehabil. 2001;80:572-7.

32. Lewis CL, Sahrmann SA, Moran DW. Anterior hip joint force increases with hip extension, decreased gluteal force, or decreased iliopsoas force. J Biomech. 2007;40:3725-31. 